\title{
Organización de contenidos e innovaciones estructurales en los diarios en línea: el caso de El Mundo
}

\author{
Content organization and structural innovations in online journals: the case of "El Mundo" (Spain)
}

\author{
Javier Merchán Sánchez-Jara, Sofia Pelosi Pelosı
}

GIR e-Lectra, Universidad de Salamanca, España. javiermerchan@usal.es; sofia.pelosi01@gmail.com

\begin{abstract}
Resumen
Se aborda un análisis comparativo de la estructura interna de los diarios de noticas entre los paradigmas impreso y digital a través de un análisis de caso. La investigación, de carácter cualitativo basada en la observación directa del fenómeno y el análisis documental, se desarrolla cotejando la versión impresa y el portal web del periódico español El Mundo. Se constatan diferencias significativas entre ambos a nivel de la estructuración y presentación de los contenidos; igualmente se confirma la consolidación de un proceso decidido de convergencia a nivel textual que revela la existencia de unidades de edición y redacción comunes para ambos medios.
\end{abstract}

Palabras clave: Diarios en línea. Medios de comunica-ción. Lectura digital. Contenidos digitales. El Mundo. España.

\section{Introducción}

Uno de los aspectos más relevantes y definitivos en los proceso de planificación de cualquier empresa o industria es la evaluación de resultados, a tenor de los datos relacionados con el cumplimiento y desarrollo de los objetivos y programas establecidos en fases previas. En el ámbito periodístico y de las industrias de la información, una parte muy relevante de estos datos giran en torno a la penetración, aceptación y consumo del producto informativo que producen, adoptándose medidas, y desarrollándose estrategias encaminadas a conseguir las cuotas más altas posibles de lectores dentro de su ámbito de acción.

La restructuración, renovación y búsqueda de nuevas formas de organización es una práctica esencial en la adaptación de las empresas a los cambios y transformaciones que se producen dentro del entorno en el que operan. El Informe de Contenidos Digitales de la Asociación Multisectorial de Empresas de Tecnologías de la Información, Comunicaciones y Electrónica (ASIMELEC, 2010) sugería que el mercado de la prensa español está viviendo una transición acelerada hacia la prensa digital tras la crisis del mercado publicitario

\begin{abstract}
A comparative analysis is done of the internal structure of newspapers between their two paradigms, print and digital. A qualitative research was developed collating the printed version and the web portal of the Spanish newspaper El Mundo. Significant differences between content structure and presentation are revealed; also, the consolidation of a strong textual convergence is confirmed, revealing the existence of common units of editing and writing for both media.
\end{abstract}

Keywords: Online newspapers. Communication media. Digital reading. Digital contents. El Mundo. Spain.

iniciada en el año 2008. En este sentido, los cambios y la dimensión de las fuerzas implicadas en el desarrollo de las nuevas de las tecnologías de la información y la comunicación en la esfera digital, surgidas en las últimas décadas, son de tal magnitud que suponen que, en cualquier caso, no solo hablemos de un espacio novedoso y renovado casi en su totalidad, si no que su derivación última es el surgimiento de un nuevo escenario paradigmático que implica que hablemos de un antes y un después.

Internet está llevando el papel hacia su fin. Así como está sucediendo con otras publicaciones (facturas, impresos efímeros, boletines oficiales, etc.), el futuro de la prensa es incierto. Su situación económica está en crisis flagrante; la productividad de los medios también. El mismo rol del periodista ante la sociedad y ante su público es vago. El siglo XXI ha inaugurado una era totalmente nueva para la comunicación global (García, 2011).

Como consecuencia de este nuevo contexto informativo, en los últimos años gran parte de estos esfuerzos reestructuradores y renovadores en la industria de la información, han estado centrados 
en adaptar los propios mecanismos de distribución del producto informativo a las fuerzas sinérgicas presentes en el desarrollo del entorno web. Este hecho ha supuesto la traslación de gran parte del capital humano perteneciente a las antiguas redacciones de los medios impresos, a la producción y edición de los propios contenidos digitales a través de las herramientas que ofrecen las nuevas tecnologías de la información y la comunicación (López y Murciano, 2012). La dimensión de estas transformaciones ha sido tan notoria y relevante que en apenas dos décadas ha supuesto la suplantación de una industria estable y consolidada durante siglos por un nuevo conjunto de estrategias comerciales en las que el modelo de negocio tradicional y su producto arquetípico, el diario impreso, se ha visto relegado a ocupar un papel de carácter subsidiario como una pieza más en un ecosistema multidimensional en el que coexisten distintos posicionamientos, y en el que a día de hoy aún surgen y se proponen nuevas vías de desarrollo y adaptación.

En el desarrollo cronológico de todas estas trasformaciones y estrategias de adaptación acontecidas en el ecosistema periodístico-informativo, hemos asistido al surgimiento de al menos tres grandes escenarios nítidamente diferenciados que permiten que hablemos de un nuevo panorama de carácter heterogéneo y multidimensional, frente al tradicional ámbito de la prensa impresa, que ha permanecido prácticamente inmutable en sus aspectos más esenciales desde prácticamente la primera mitad del siglo XX (Moreno, 2004).

En este sentido es necesario destacar como la irrupción de la distribución de contenidos a través de la web ha propiciado, en primer lugar, el surgimiento de nuevos medios (inexistentes con anterioridad en el ámbito impreso), que por su especial carácter novedoso, la singularidad de su línea editorial, o un marcado perfil aperturista en relación a la interacción con sus usuarios, se han convertido en grandes medios de comunicación en un espacio de tiempo relativamente muy corto. El caso del Huffinton Post, es quizás unos de lo ejemplos mas paradigmáticos en este sentido (Doyle, 2013). De manera paralela y antagónica, muchos de los diarios y medios tradicionales preexistentes en el ámbito analógico han ido perdiendo representatividad dentro del espectro de los medios de comunicación, y han estado abocados a su desaparición y cierre; o, en el mejor de los casos, a continuar únicamente con la distribución de la versión en línea. Un tercer gran grupo de diarios, quizás el más numeroso y representativo de todos en el sentido de su inserción dentro de los grandes holdings editoriales, es el de aquellos que mantienen su edición impresa, y que han desarrollado de manera paralela un versión en línea en torno a toda la serie de nuevas potencialidades y posibilidades que el nuevo medio brinda, conformándose como estructuras multiplataforma implementadas en relación a distintos medios y modalidades de acceso y distribución. Por todo ello, podemos afirmar que surgen nuevas posibilidades de acceso a la información textual, que representan distintas respuestas a un conjunto de necesidades y perfiles de lector tan heterogéneos como diversificados (Ramírez, 2005).

Por un lado surge la posibilidad de acceso a la versión digitalizada (facsímil) de la versión impresa también conocida como e-Papers (Goyanes; Dürrenberg, 2014), mediante la modalidad de suscripción por un espacio de tiempo determinado, o a través del pago unitario, como forma de acceso a un número concreto. Esta modalidad , además, se articula en torno a dos posibilidades claramente diferenciadas.

Por un lado se posibilita la suscripción o el acceso unitario a través de los grandes portales de acceso de estos contenidos, kioscos digitales y plataformas como Orbyt, o a través de las propias aplicaciones dedicadas que cada medio o publicación dispone como forma de acceso principal a los contenidos a través dispositivos móviles. Dentro de este ámbito debemos resaltar que algunos medios, como es el caso del diario El Mundo, desarrollan de igual manera aplicaciones diferenciadas para el acceso a la versión en línea gratuita, o para el acceso a la versión impresa (facsímil digital) mediante las ya citadas opciones de suscripción o pago por ítem.

Pero sin duda la categoría más representativa de los nuevos diarios digitales, se corresponde con lo que podemos denominar como versiones streaming, diarios en línea o en su denominación más genérica, pagina web informativa (Legorburu, 2012). Estos portales web se caracterizan por permitir el acceso libre y gratuito a las noticias e informaciones que el medio produce o recoge, así como a gran cantidad de otro tipo de contenidos y servicios amparados bajo el auspicio editorial de la propia institución. Además, en un estadio intermedio entre la versión impresa de pago y el portal web, estos servicios se complementan mediante la disposición de plataformas de carácter híbrido, y acceso a través del modelo freemium mediante el pago por suscripción, que permite el acceso a toda una serie de contenidos y de prestaciones adicionales, que de una manera u otra incorporan un valor añadido con respecto a los contenidos genéricos alojados en el propio diario. En la actualidad, en el diario El Mundo esta modalidad de acceso está disponible a través de la plataforma 
Orbyt.es y ofrece servicios como el acceso a las 20 ediciones regionales del diario, la consulta de la hemeroteca, así como otro tipo de funcionalidades y herramientas, un mayor grado de interacción con los contenidos, una mejora sustancial en la experiencia de usuario, o la disposición de foros y espacios virtuales para la comunicación con los propios responsables de la institución.

En España, en el momento actual, y aún cuando los modelos de distribución y acceso a los contenidos digitales se configuran como un ámbito en continuo desarrollo y evolución, la mayor parte de los grandes medios cuentan con un espacio web de acceso libre y gratuito a través del cual se puede acceder a la práctica totalidad de los contenidos que publican (1). Al margen de las corrientes globalizadoras bajo las que surgen y se implantan la mayoría de las nuevas prácticas en el ámbito de la información y la comunicación en la esfera digital, en España la generalización y consolidación de esta modalidad de acceso libre y gratuito a la totalidad de los espacios en línea de los grandes diarios, ha estado marcada de forma ineludible, por los resultados, (tan llamativos como esclarecedores), cosechados por los dos gigantes de la prensa española en su implantación en el espacio web a tenor de las estrategias antagónicas que cada uno de ellos puso en liza en su debut en el medio digital. Mientras que le diario El País optó por mantener el acceso a su portal web a través del modelo por suscripción desde el año 1996 hasta 2005, como medio de preservar los derechos de autoría y propiedad industrial de sus contenidos impresos, El Mundo eligió, de manera más clarividente, ofrecer la versión impresa (en formato digital) mediante el modelo de suscripción, y apostar por el acceso gratuito a los contenidos alojados en el portal web. Independientemente de que unos años después El País decide abandonar sus lineamientos primigenios para apostar por estrategias de distribución similares a las de El Mundo, como reconocimiento tácito de una posicionamiento comercial fallido que únicamente fue capaz de filiar a 45000 lectores en el ámbito nacional, este hecho provocó la consolidación, de manera concurrente, del despegue del diario El Mundo como el medio más accedido vía web. Esta coyuntura está relacionada de forma directa, sin lugar a dudas, con la consolidación de del propio diario El Mundo como publicación impresa más leía a nivel nacional en la primera década del siglo XXI (Cerezo, 2009).

En el fondo de toda esta cuestión subyace una realidad incuestionable: el escaso tráfico de visitas en los medios cerrados, o de suscripción, provoca una disminución tan significativa en las aportaciones generadas por publicidad, que conduce, en definitiva, a que la suma de ingresos provenientes de suscripción, y los que se generan por publicidad, son sensiblemente menores a los que la propia industria publicitaria aporta en los medios abiertos que soportan un mayor número de visitas (Goyanes, 2013).

Todas estas consideraciones representan el fenómeno catalizador y responsable de la precipitación de una serie de cambios muy sensibles a todos los niveles en una industria asentada, fijada y con estabilidad reconocida desde hace décadas a nivel mundial; cambios que transitan desde la organización interna de las propias instituciones productoras, al posicionamiento estratégico en relación al modelo de negocio o a la propia formación y nivel de competencia tecnológica exigible al capital humano que las integra (Rojo, 2005); cambios que afectan de igual manera al propio producto informativo; su forma de acceso o la arquitectura en torno a la cual se estructura su presentación; cambios que en cualquier caso seguirán produciéndose bajo la premisa de que el futuro de los medios de comunicación está ligado de manera inevitable a un ámbito digital que plantea un horizonte repleto de interrogantes.

\section{Objetivos}

El objetivo de este trabajo es establecer patrones y regularidades que permitan fijar una estructura básica en la edición impresa que posibilite una comparación directa con la arquitectura y organización de los contenidos en el portal web El Mundo.es.

\section{Metodología}

Para la consecución del objetivo se plantean distintas categorías de presentación de los contenidos dentro de la estructura del propio medio; cuatro para la versión en línea -diario en línea, webs asociadas, suplementos y servicios (Figura 2)_; dos para la versión impresa - diario y suplementos- (Figura 1).

Se establece la sección y su desarrollo (subsecciones, partes, etc.) como unidad taxonómica para la comparación y análisis de la estructura de ambas plataformas. Se considera como unidad de análisis de las categorías de observación de cada una de las versiones: la versión impresa de la edición Madrid, y el diario en línea de acceso libre y gratuito. En ambos casos se analizan y cotejan todas las ediciones y ejemplares publicados en un periodo de observación que abarca un arco cronológico que abarca tres semanas desde el lunes 15 de febrero hasta el domingo 6 de marzo 
de 2016, constituyendo una muestra de 21 unidades de observación.

\section{Resultados}

\subsection{Arquitectura del espacio informativo}

La migración de la prensa en el papel a plataformas digitales ha propiciado cambios tanto en la arquitectura y la organización de los contenidos, como en los aspectos relacionados con las unidades redaccionales.

La diferenciación más genérica entre los diarios impresos y las páginas web informativas (Legorburu, 2012) se sustancian en que los primeros se conforman como fotos fijas de un conjunto de realidades, es decir, como instantáneas de los eventos más relevantes dentro de un contexto socio-cultural y territorial concreto, que son susceptibles de ser de interés a un determinado grupo más o menos heterogéneo de personas; en este sentido los diarios impresos se conforman como nuestro diario. Nos encontramos ante un concepto autorial de la composición informativa, de carácter cerrado, estático y circunscrito únicamente al plano de la actualidad más inmediata.

Por su parte, el medio digital posibilita la representación de la información de manera análoga a como los hechos acontecen en la realidad. La noticia se incorpora al medio de manera concurrente a su desarrollo real; y su posicionamiento en la representación virtual de la página permite reproducir y reflejar, de manera intuitiva, aspectos como su relevancia informativa, la distancia en el tiempo desde su aparición hasta el momento presente, o posibilitar, en los casos más notorios, el propio seguimiento en directo del transcurso de los acontecimientos a través de retransmisiones multimedia. Todas estas consideraciones implican que las estructuras que vertebran los nuevos diarios digitales estén inspiradas en la voluntad de mostrar, ofrecer y facilitar la localización de la información de manera clara y rápida.

En la versión impresa la disposición de las secciones, o artículos está relacionada con la necesidad de mostrar un relato acabado, en torno a criterios como la relevancia informativa en su ámbito, los alineamientos ideológicos de la institución, o la voluntad de ofrecer una lectura variada, y atractiva. Por su parte, en el medio digital muchos de estos condicionantes pasan a un segundo plano en función de otras serie de cuestiones de mayor importancia: el posibilitar que cada usuario configure su propio itinerario informativo, en un contexto más globalizado y descontextualizado; o la necesidad de configurar un espacio que integre los distintos medios disponibles: audio, video, fotografía, etc.

En contraposición a la disposición de los contenidos en la versión impresa - que responde a la necesidad de construir un relato ordenado-, la versión en línea debe encarar condicionantes mucho más numerosos, por lo que su diseño resulta una tarea crítica para la configuración del medio. Estos espacios no solo deben de resultar intuitivos y fáciles de usar; deben, además, constituirse como archivos que permitan la organización de toda la información para poder recuperarla de forma eficiente y contextualizada con el resto de contenidos. Esta interrelación de contenidos exige el desarrollo de un sistema de categorías, etiquetas e hiperenlaces internos, que evite la redundancia y el ruido informativo producido por la duplicidad de contenidos en distintas secciones.

\subsection{Organización, estructura y presentación} de los contenidos

En ambos casos la información se clasifica en torno a una serie de divisiones principales que se muestran como categóricas y estables (Figuras 1 y 2) y a partir de ellas se desarrollan los siguientes subniveles que permiten adecuar cada contenido a un epígrafe lo más específico posible. Este desarrollo taxonómico se reduce a uno o dos niveles en la versión impresa, mientras que el diario en línea pueden llegar hasta 4 ó 5 . Este hecho permite una mayor granularidad en el acceso a los contenidos, pero implica la sensación de encontrarse en un entramado laberíntico, ya que la interconexión de secciones y contenidos permite acceder a ellos por distintas rutas. En la versión impresa, la estructura se muestra siempre como reconocible, a pesar de que existen variaciones en el orden y aparición de alguna de las secciones, que dependen de los propios contenidos y de los días de publicación (Figura 1).

En términos generales, estas categorías tienen un carácter menos específico: por ejemplo, la sección de deportes se conforma en torno a reportajes de actualidad enmarcados bajo la sección genérica de deportes y sin subdivisiones para las distintas especialidades. En el caso de la edición impresa únicamente se recurre a estas subsecciones en las noticias de carácter coyuntural que no incorporan alusiones fotográficas de la disciplina. Por otro lado, mientras que en el diario en línea las subdivisiones taxonómicas están siempre presentes, en la versión impresa estas únicamente se reflejan asociadas a informaciones y reportajes de carácter especial o monográfico. 
ElMundo;

edicción impresa viemes 4 de marzo 2016

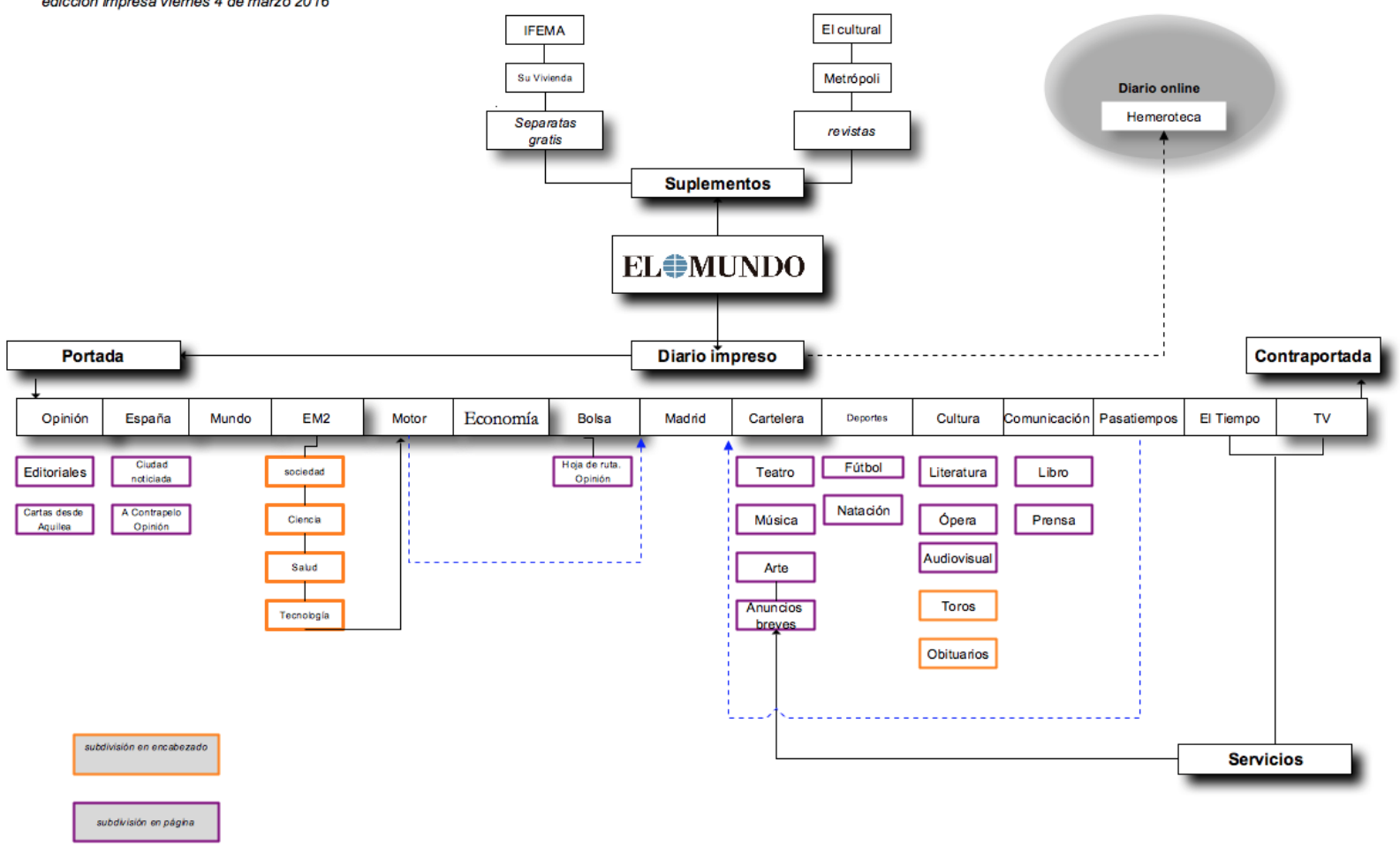

Figura 1. Categorías y secciones en diario impreso

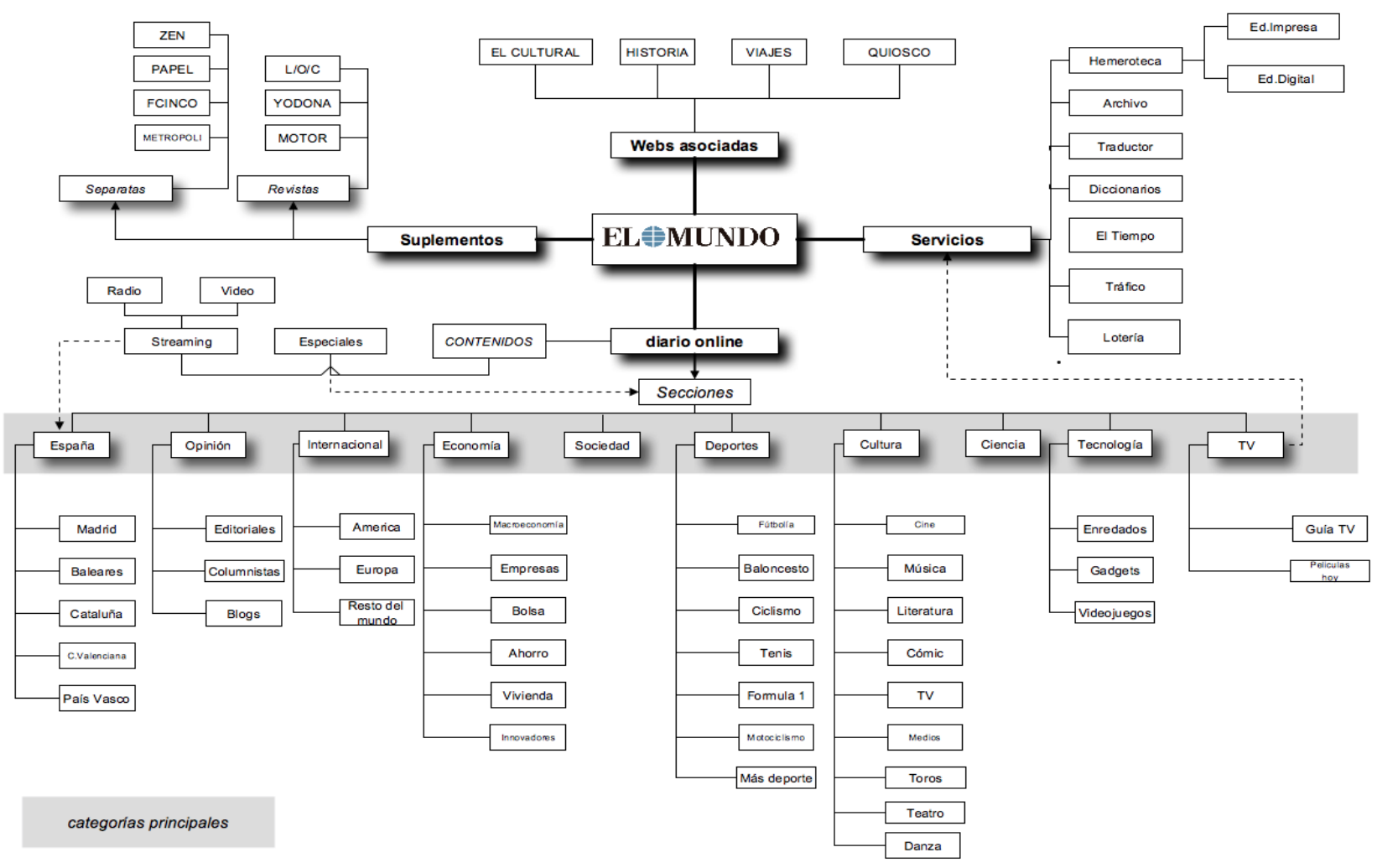

Figura 2. Arquitectura de contenidos en El Mundo diario en línea 

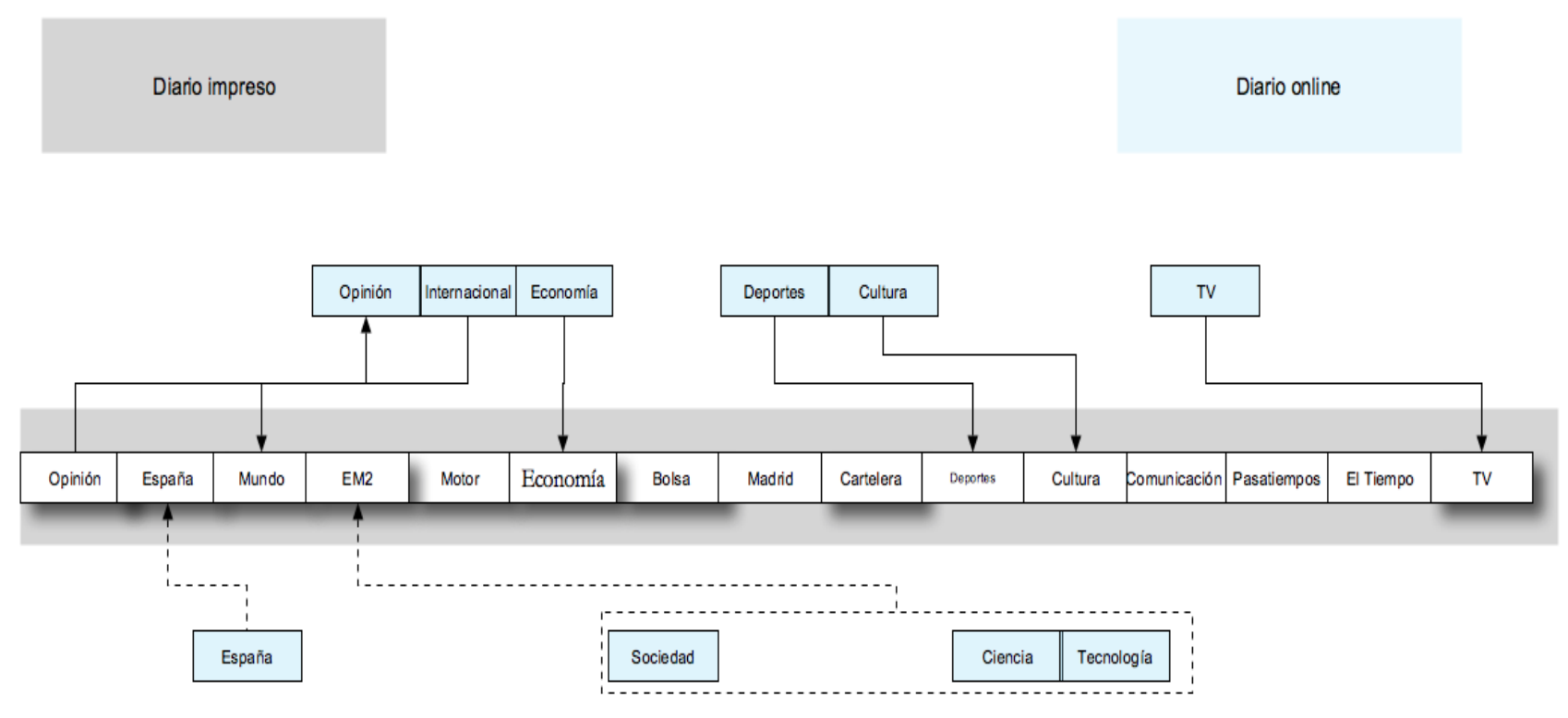

Orden de presentación de secciones principales en diario impreso y homepage de diario online

Figura 3. Secciones y categorías básicas en edición impresa y homenaje del diario en línea

\subsection{Epígrafes y denominaciones de secciones}

Existen ciertas diferencias en torno al número, posición o denominación de las secciones entre ambas modalidades. Mientras que la versión impresa incorpora 15 secciones principales, la digital se configura en torno a 10, en gran parte debido a que las secciones en línea presentan una serie de subdivisiones internas que no están presentes en la versión impresa, (Figura 2 y 3). En este sentido la edición impresa refleja una estructura de carácter más horizontal, mientras que la versión en línea muestra una organización de carácter arborescente.

La posición que estas grandes categorías ocupan en el espacio informativo, también difieren de manera sensible (Figura 2), si bien es cierto que el acceso no secuencial a los contenidos en el medio en línea no tiene las mismas consideraciones que en la versión impresa. Otro aspecto a destacar, es que la versión impresa incorpora una especie de sección central denominada EM2 que agrupa algunas categorías de la versión en línea como sociedad, ciencia, o tecnología.

Es necesario reseñar también diferencias en el tipo de contenidos que se asocian bajo un mismo epígrafe. La sección motor del diario en línea incorpora contenidos de carácter divulgativo en torno a el mundo del automóvil en general, mientras que la misma sección en la versión impresa tiene un carácter relacionado con las implicaciones económicas del sector del automóvil.

\subsection{Estructura y carácter de los contenidos}

El cambio de soporte ha impuesto sus condiciones y el artículo periodístico ha aceptado el reto de adaptarse a ellas, integrando la tradicional estructura con aspectos propios del texto digital como el hipertexto, el uso de audio y vídeo, la interactividad o la actualización en tiempo real.

La comparación de contenidos en los diferentes soportes refleja cómo en la prensa en línea el artículo se convierte en una estructura más elaborada que incorpora el cuerpo del texto y una serie de elementos paratextuales, que van desde elementos multimedia, hipervínculos que apuntan a otras noticias relacionadas, espacios para la discusión, o herramientas de lectura social. Estos nuevos textos-documentos abren las puerta a distintos niveles de interacción y profundización con la información y a múltiples posibilidades de lectura (Alcoba y Freixas, 2009). Todos estos elementos, junto al carácter no lineal de los diarios en línea, implican que el acceso al contenido requiera la intervención intencionada del lector que 
necesita discriminar entre los contenidos disponibles. Por todo ello, en este tipo de medios se impone la presentación de la noticia a través de estrategias que permitan aportar el mayor nivel de entropía en la información; la tendencia más marcada conduce a disposiciones que en la mayoría de los casos únicamente consisten en el título y una imagen alusiva que incorpora el propio reproductor de video, implicando que se pueda acceder al contenido a través del objeto multimedia sin tener que recurrir a la lectura del texto.

\subsection{Diferencias en los aspectos formales y de diseño}

En los últimos tiempos los medios de comunicación han implementado estrategias de convergencia entre los recursos informativos del impreso y los relacionados con el medio digital; estrategias que pretenden la unificación de las unidades de redacción y producción de contenidos como medio para economizar costes (Salaverría; Negredo, 2008), pero también agilizar procesos en un contexto en el que la actualización de los contenidos es esencial.

Por todo ello, nos encontramos en una situación en la que las unidades de redacción y producción elaboran contenidos que finalmente son destinados de manera indistinta a ambos medios. Un escrutinio en el contenido de los artículos en ambas versiones, revela que estos son prácticamente idénticos en una gran mayoría de los casos, y que las diferencias, siempre de carácter mínimo, responden a una serie de tipologías que pueden ser sintetizadas en los siguientes grupos:

- Variaciones en el cuerpo del texto.

- El cuerpo del texto es idéntico, y se presentan diferencias en la redacción del titular o subtitulares. Estos ocurren generalmente en contenidos de máximo interés en los que el titular se ha actualizado en la versión web.

- El texto es idéntico en ambas versiones pero una de ellas presenta el contenido recortado en algunas líneas o párrafos, es decir textos idénticos pero adaptados.

- Artículos editados en extensión y estilo. Se trata generalmente de textos adaptados a la versión en línea en los que se suprimen fragmentos o se modifica la redacción.

- Noticias en las que el texto es diferente en su gran mayoría; y únicamente comparten los titulares, las entradillas o algún párrafo.

Si las diferencias en el cuerpo del texto son muy poco significativas, no ocurre lo mismo con su disposición y los elementos paratextuales, en los que se pueden constatar diferencias relevantes.
Al tradicional modelo compositivo de las noticias en prensa escrita, consistente en un texto acompañado de una o varias fotografías, se unen ahora otras formas de presentación en virtud de las nuevas posibilidades del medio digital. La mayoría de ellas están influenciadas por la presencia de elementos multimedia que ya no solo complementan la información textual, sino que en muchas ocasiones conforman per se el contenido informativo. Así, es frecuente el uso de reportajes fotográficos donde el texto apenas representa información de carácter descriptivo, y en los que toda la carga semántica está implícita en la propia imagen.

Más disruptivos son aún los contenidos de carácter videográfico que permiten la reproducción del propio hecho informativo de manera real y cuyas potencialidades son de tal magnitud, que la mayoría de los medios han adaptado sistemas de retransmisión streaming para el seguimiento en riguroso directo. Todos los contenidos multimedia son materiales y formas de presentación exclusivos del medio digital, por lo que, a nivel de presentación y forma de comunicación, asistimos a un proceso de divergencia.

El otro gran espacio de diferenciación está relacionado con la lectura social, el nivel de participación y los roles del lector, pues el medio digital permite un nuevo paradigma en la participación de la comunidad lectora.

\section{Conclusiones}

La mayoría de los medios de prensa escrita mantienen en la actualidad portales web de carácter multidimensional. Estos se disponen en torno a una estructura organizativa muy desarrollada, que constituye la vía principal para la puesta a disposición y acceso a los contenidos que producen.

Nos encontramos en un estadio de evolución de este tipo de modelos de negocio que permite que la publicidad se muestre transparente y que únicamente aparezca cuando se accede a los recursos de carácter multimedia (videos y streaming), como peaje para el acceso a contenidos que representan un plus con respecto a los presentes en la prensa tradicional.

Si en un primer estadio el pago por suscripción garantizaba el acceso a contenidos más elaborados, en la actualidad se han implementado procesos de convergencia en el aspecto meramente textual que unifican los equipos de edición y redacción que elaboran los contenidos para ambos medios. 
El cotejo de los contenidos en ambas versiones permite afirmar que no existen diferencias significativas a nivel semántico entre los contenidos presentes en uno u otro medio. En una gran mayoría de los caso los textos son idénticos de principio a fin, y las únicas variaciones se corresponden con la supresión de pequeños párrafos, o en cambios en la redacción del titular o la entradilla del artículo.

Las diferencias más sensibles se constatan en aspectos como la propia estructura organizativa de la edición, en el aparato paratextual que acompaña al cuerpo del artículo o en las nuevas formas de presentación del texto, como son los enlaces hipertextuales.

En cualquier caso, aunque en el periódico impreso se puede acceder a los contenidos de manera parcial o intencional en relación a capítulos o contenidos concretos, la propia estructuración se corresponde con la intención editorial de dotar a la obra de un sentido y jerarquía concreta que se pone de relieve en torno a una idealizada lectura lineal del documento.

En el diario en línea, se impone una lectura más focalizada y selectiva, ello se debe a que en este medio los contenidos se conforman como estructuras estancas que en ningún caso dan paso al siguiente contenido de manera automática o preestablecida.

De esta forma, en este nuevo paradigma es de vital importancia la intervención del lector, que actúa como el propio organizador o editor de su versión del diario, dada la necesidad de seleccionar cada una de las noticias que se desean leer. La posibilidad de elección a nivel de multimedialidad, hipertextualidad o rango cronológico permite que los espacios informativos web se constituyan como la herramienta para la configuración de lo que algunos autores refieren como "mi diario" (Schoenbach, 2005).

\section{Notas}

(1) Los facsímiles digitales de la edición impresa y los contenidos digitalizados de sus respectivas hemerotecas son una excepción notoria que queda excluida de esta tendencia.

\section{Referencias}

Acevedo, Mónica. (2005). La personalización en la prensa digital española: una excusa rentable. // Zer: Revista de estudios de comunicación = Komunikazio ikasketen aldizkaria. 18:1 (2005) 143-157.

Alcoba, Santiago; Freixas, Margarita (2009). La prensa digital: condiciones y lengua. // Sarmiento, Ramón; Vilches, Fernando.(coord.). La Calidad Del Español En La Red. Nuevos Usos de La Lengua En Los Medios Digitales. Barcelona: Ariel (Col. Fundación Telefónica), 2009. 27-57.
Alonso, Pedro. (2011). Prensa impresa o prensa digital. // Cuenta y razón. 20:1 (2011) 31-36.

Asociación Multisectorial de Empresas de Tecnología de La Información, Comunicación y Electrónica (2010). Informe de la industria de contenidos digitales. Madrid: ASIMLEC. http://www.osimga.gal/export/sites/osimga/gl/documentos/d/2010_10_06_ASIMELEC_informe2010_contenidos_digitales.pdf (2016-09-20).

Cerezo, Julio. (Ed.). (2009). La revolución de la prensa digital. // Evoca Comunicación e Imagen. (2009). http://www.evocaimagen.com/cuadernos/cuadernos1.pdf (2016-09-21).

De Nicolás, Sara. (2004). Ventajas y desventajas de la prensa digital en relación a la prensa impresa. // Tonos digital: Revista electrónica de estudios filológicos. 8:4, (2004)

Doyle, Gillian. (2013). Re-invention and survival: newspapers in the era of digital multiplatform delivery.// Journal of Media Business Studies. 10:4 (2013) 1-20.

Goyanes, Manuel. (2013). Estrategias y modelos de negocio: aclaración de conceptos y terminología de la prensa en Internet. // Estudios sobre el Mensaje Periodístico. 19:1 (2013) 419-431.

Goyanes, Manuel; Dürrenberg, Catherina. (2014). A Taxonomy of Newspapers based on Multi-Platform and Paid Content Strategies: Evidences from Spain. // International Journal on Media Management. 16:1 (2014) 27-45.

Legorburu, José María (2012). Convergencia de medios: Nuevos desafíos para una comunicación global. Madrid: Fundación Universitaria San Pablo, 2012.

Rabadán, Pablo; Martínez, Marcial. (2012). Retos del periodismo ante el nuevo escenario digital. // adComunica: revista científica de estrategias, tendencias e innovación en comunicación. 4:1 (2012) 17-19.

Salaverría, Ramón; Negredo, Samuel. (2008). Periodismo integrado: convergencia de medios y reorganización de redacciones. Barcelona: Sol90, 2008.

Schoenbach, Klaus. (2005). Research Note: Online and Print Newspapers: Their Impact on the Extent of the Perceived Public Agenda. // European Journal of Communication, $20: 2$ (2005) 245-258.

Villada, Pedro Antonio. (2006). Prensa y convergencia tecnológica: claves para la definición de un nuevo modelo de negocio periodístico en la Era Digital. // Zer: Revista de estudios de comunicación $=$ Komunikazio ikasketen aldizkaria. 20:1 (2006) 411-424.

Enviado: 2016-04-01. Segunda versión: 2016-09-25. Aceptado: 2016-10-09. 